\title{
Using polarimeter and Stokes parameters for measuring linear birefringence and diattenuation properties of optical samples
}

\author{
P. C. Chen, Y. L. Lo* \\ Department of Mechanical Engineering, National Cheng Kung University, Tainan, 701, Taiwan \\ *loyl@mail.ncku.edu.tw
}

\begin{abstract}
A technique is proposed for measuring the linear birefringence and linear diattenuation of an optical sample using a polarimeter. In the proposed approach, the principal axis angle $(\alpha)$, phase retardance $(\beta)$, diattenuation axis angle $\left(\theta_{d}\right)$, and diattenuation $(D)$ are derived using an analytical model based on the Mueller matrix formulation and the Stokes parameters. The dynamic measurement ranges of the four parameters are shown to be $\alpha=0 \sim 180^{\circ}, \beta=0 \sim 180^{\circ}, \theta_{d}=0 \sim 180^{\circ}$, and $D=0 \sim 1$, respectively. Thus, full-range measurements are possible for all parameters other than $\beta$. In this study, the proposed methodology does not require the principal birefringence axes and diattenuation axes to be aligned. In addition, the linear birefringence and linear diattenuation properties are decoupled within the analytical model, and thus the birefringence properties of the sample can be solved directly without any prior knowledge of the diattenuation parameters.
\end{abstract}

\section{Introduction}

Diattenuation is the property of an optical system whereby the intensity transmittance of the exiting beam depends on the polarization state of the incident beam [1-3]. It has been shown that the diattenuation of an optical sample can be calculated directly from the elements of the corresponding Mueller matrix; namely a matrix containing information about all of the polarization properties of the system [3]. In 1990, Chenault and Chipman [4] measured the diattenuation and birefringence of IR samples using a spectropolarimeter. A few years later, the same authors used a rotating sample spectropolarimeter to measure the birefringence spectra of three ferroelectric liquid crystals, namely 764E, SCE4 and SCE9, in the infrared wavelength region from 2.5 to $16.5 \mu \mathrm{m}$ [5]. In 1993, the same group presented a technique for measuring the diattenuation and birefringence spectra of a sample rotated between two stationary linear polarizers in an IR-spectrometer [6]. In [4-6], the optical parameters were solved using an analytical approach based on the first three terms of the FourierBessel expansion of optical intensity. However, in [4-6], an assumption was made that the principal birefringence and diattenuation axes were aligned. Furthermore, the linear birefringence and linear diattenuation parameters were coupled within the corresponding analytical models. In addition, solving the sample parameters with a Fourier-based scheme is intended to simplify the measurements and data analysis is a bit more involved in the Fourier-transform case. Bueno and Artal $[7,8]$ used a double-pass polarimeter to investigate the ocular diattenuation phenomenon using

This is an Open Access article distributed under the terms of the Creative Commons Attribution-Noncommercial License 3.0, which permits unrestricted use, distribution, and reproduction in any noncommercial medium, provided the original work is properly cited. 
a Mueller matrix formulation. Todorovic et al. [9] investigated the diattenuation of biological samples using an optical coherence tomography technique and a Mueller matrix formulation. Huang and Knighton [10] measured the diattenuation spectrum of the retinal nerve fiber layer using a multispectral imaging micropolarimeter. However, in [7-10], they only measured the diattenuation of material.

In recent years, several methodologies have been presented for measuring the linear birefringence and diattenuation properties of optical samples using near-field scanning optical microscopy (NSOM) techniques. For example, Higgins et al. [11] presented a method for measuring the diattenuation of mesoscopic crystals in which an electro-optic modulator and a quarter-wave plate were used to modulate the polarization of the light was used to extract the sample parameters. Campillo and Hsu [12] measured the birefringence and diattenuation of SiN membranes using a SNOM in which the polarization of the input light was adjusted using a photo-elastic modulator (PEM) and the detected signal was processed using a Fourier analysis scheme. Fasolka et al. [13] and Goldner et al. [14-16] adopted a similar approach to measure the local optical properties of photonic block copolymers and isotactic polystyrene crystallites, respectively. In contrast to previous methods, the schemes presented in [11-16] assumed that the principal axes of birefringence and diattenuation were not necessarily aligned in the optical sample.

Accordingly, this study presents a straightforward method for measuring the local birefringence and diattenuation properties of an optical sample using a polarimeter and a Mueller matrix formulation. In the proposed approach, we use Stokes parameters and change the state of polarization of a light to measure the optical sample. It is shown that the proposed methodology enables full range measurements of the principal axis angle, diattenuation axis angle and diattenuation, respectively. In addition, the linear birefringence and linear diattenuation parameters are decoupled in the analytical model. Thus, the birefringence parameters can be solved directly without any prior knowledge of the diattenuation parameters. The concept of characteristic parameters will be used to prove the derived algorithm for measuring the local effective birefringence and diattenuation properties in tested samples such as a baked polarizer and a composite sample that includes a baked polarizer in series with a quarter-wave plate. Two cases are discussed when considering the diattenuation axis of a baked polarizer is aligned or not aligned to the principal axis angle of a quarter-wave plate.

\section{Basic measuring system and methods}

\subsection{Basic Stokes parameter method}

The state of polarization of a light beam can be completely described by the Stokes vector $S=\left\{S_{0}, S_{1}, S_{2}, S_{3}\right\}^{T}$. Any optical component which changes the polarization state of a light ray can be modeled by a $4 \times 4$ Mueller matrix. The inner product of this Mueller matrix $M$ and the Stokes vector $\hat{S}$ representing the polarization state of the incident light yields a Stokes vector $S$ which describes the polarization state of the light exiting the optical sample [17]. According to the Stokes-Mueller formulation, the Stokes vector of the output light can be derived as $S=M \hat{S}$, where $\hat{S}=$ $\left\{\hat{S}_{0}, \hat{S}_{1}, \hat{S}_{2}, \hat{S}_{3}\right\}^{T}$ is Stokes vector of the input light.

In this study, the samples of interest are assumed to have both linear birefringence and linear diattenuation properties. According to [18], the Mueller matrix for a linear birefringent material such as a wave plate or retarder can be expressed as

$$
M_{l b}=\left(\begin{array}{cccc}
1 & 0 & 0 & 0 \\
0 & \cos (4 \alpha) \sin ^{2}(\beta / 2)+\cos ^{2}(\beta / 2) & \sin (4 \alpha) \sin ^{2}(\beta / 2) & \sin (2 \alpha) \sin (\beta) \\
0 & \sin (4 \alpha) \sin ^{2}(\beta / 2) & -\cos (4 \alpha) \sin ^{2}(\beta / 2)+\cos ^{2}(\beta / 2) & -\cos (2 \alpha) \sin (\beta) \\
0 & -\sin (2 \alpha) \sin (\beta) & \cos (2 \alpha) \sin (\beta) & \cos (\beta)
\end{array}\right)
$$


where $\alpha$ is the principal angle of the slow axis and $\beta$ is the phase retardance. Similarly, the Mueller matrix for a linear diattenuation material can be expressed as

$$
M_{l d}=\left(\begin{array}{cccc}
\frac{(u+v)}{2} & \frac{\cos \left(2 \theta_{d}\right)(u-v)}{2} & \frac{\sin \left(2 \theta_{d}\right)(u-v)}{2} & 0 \\
\frac{\cos \left(2 \theta_{d}\right)(u-v)}{2} & \frac{(\sqrt{u}+\sqrt{v})^{2}}{4}+\frac{\cos \left(4 \theta_{d}\right)(\sqrt{u}-\sqrt{v})^{2}}{4} & \frac{\sin \left(4 \theta_{d}\right)(\sqrt{u}-\sqrt{v})^{2}}{4} & 0 \\
\frac{\sin \left(2 \theta_{d}\right)(u-v)}{2} & \frac{\sin \left(4 \theta_{d}\right)(\sqrt{u}-\sqrt{v})^{2}}{4} & \frac{(\sqrt{u}+\sqrt{v})^{2}}{4}-\frac{\cos \left(4 \theta_{d}\right)(\sqrt{u}-\sqrt{v})^{2}}{4} & 0 \\
0 & 0 & 0 & \sqrt{u v}
\end{array}\right)
$$

where $\theta_{d}$ is the diattenuation axis angle and $u$ and $v$ are the transmittances of the optical sample for light rays polarized parallel and perpendicular to the diattenuation axis, respectively [14]. The diattenuation of the sample is given by $D=(u-v) /(u+v)$.

Assume that the sample of interest comprises an optical material with linear birefringence properties positioned in front of a second optical material with linear diattenuation properties [14]. As a result, the composite sample has four parameters of interest, namely the principal axis angle $(\alpha)$, the phase retardance $(\beta)$, the diattenuation axis angle $\left(\theta_{d}\right)$, and the diattenuation $(D)$. The output Stokes vector $S_{c}$ of the composite sample can be obtained as

$$
S_{c}=\left[\begin{array}{c}
S_{0} \\
S_{1} \\
S_{2} \\
S_{3}
\end{array}\right]_{c}=\left[M_{l d}\right]\left[M_{l b}\right] \hat{S}_{c}=\left(\begin{array}{llll}
m_{11} & m_{12} & m_{13} & m_{14} \\
m_{21} & m_{22} & m_{23} & m_{24} \\
m_{31} & m_{32} & m_{33} & m_{34} \\
m_{41} & m_{42} & m_{43} & m_{44}
\end{array}\right)\left(\begin{array}{c}
\hat{S}_{0} \\
\hat{S}_{1} \\
\hat{S}_{2} \\
\hat{S}_{3}
\end{array}\right)_{c}
$$

where the notation $c$ indicates different input polarization states (i.e. $0^{\circ}, 45^{\circ}, 90^{\circ}, 135^{\circ}$, and righthand circular). The Mueller matrix elements for the two optical materials within the composite sample $m_{11}, m_{14}, m_{21}, m_{24}, m_{31}, m_{34}, m_{41}, m_{42}, m_{43}, m_{44}$ can be used to solve the output Stokes parameters from various input polarization states. It should be noted that the complex terms in $m_{12}$, $m_{13}, m_{22}, m_{23}, m_{32}$, and $m_{33}$ are not used to solve the effective optical parameters of an optical fiber.

As described in the following sections, given knowledge of the input polarization state and the measured values of the output Stokes parameters, the Mueller matrix elements enable the four parameters of interest to be inversely derived.

\subsection{Determination of linear birefringence and linear diattenuation properties}

In the method proposed in this study, the linear birefringence properties of the composite sample, i.e., $\alpha$ and $\beta$, are extracted from the output Stokes vectors corresponding to three different input polarization states, namely two linear polarization states, i.e., $\hat{S}_{0^{\circ}}=[1,1,0,0]$ and $\hat{S}_{45^{\circ}}=[1,0,1,0]$, and one right-hand circular polarization state, i.e., $\hat{S}_{R H C}=[1,0,0,1]$. Therefore, the principal axis angle of the linear birefringent material which its range extends from $0 \sim 180^{\circ}$ can be obtained as

$$
2 \alpha=\tan ^{-1}\left(\frac{-S_{0^{\circ}}\left(S_{3}\right)}{S_{45^{\circ}}\left(S_{3}\right)}\right)
$$

The retardance which its measurable range is from $0 \sim 180^{\circ} \mathrm{can}$ be obtained as

$$
\beta=\tan ^{-1}\left(\frac{S_{45^{\circ}}\left(S_{3}\right)}{\cos (2 \alpha) \cdot S_{R H C}\left(S_{3}\right)}\right)
$$

When determining the linear diattenuation properties of the composite sample, two additional linearly polarized input lights are chosen, namely $\hat{S}_{90^{\circ}}=[1,-1,0,0]$ and $\hat{S}_{135^{\circ}}=[1,0,-1,0]$, 
respectively. The diattenuation axis angle which its measurable range is from $0 \sim 180^{\circ}$ can be obtained as

$$
2 \theta_{d}=\tan ^{-1}\left(\frac{S_{45^{\circ}}\left(S_{2}\right)+S_{135^{\circ}}\left(S_{2}\right)}{S_{0^{\circ}}\left(S_{1}\right)+S_{90^{\circ}}\left(S_{1}\right)}\right)
$$

Having determined the value of the diattenuation axis $\theta_{d}$, the diattenuation $D$ can be obtained as

$$
D=\frac{u-v}{u+v}=\frac{S_{0^{\circ}}\left(S_{1}\right)+S_{90^{\circ}}\left(S_{1}\right)}{\cos \left(2 \theta_{d}\right) \cdot\left[S_{0^{\circ}}\left(S_{0}\right)+S_{90^{\circ}}\left(S_{0}\right)\right]}
$$

\section{Analytical results}

In this section, the ability of the proposed analytical model to extract the four effective optical parameters of interest over the measurement ranges defined in the previous section is verified using a simulation technique.

In evaluating the ability of the proposed method to extract the principal axis angle $(\alpha)$, the phase retardance $(\beta)$, the diattenuation axis angle $\left(\theta_{d}\right)$, the diattenuation $(D)$ of the composite sample, respectively. Figure $1(\mathrm{a}),(\mathrm{b}),(\mathrm{c}),(\mathrm{d})$ compares the value of $\alpha$ with the input value over the range $0 \sim$ $180^{\circ}$, the values of $\beta$ with the input values over the full range of $0 \sim 360^{\circ}$, the value of $\theta_{d}$ over the full range of $0 \sim 180^{\circ}$, all values of $D$ in the range $0 \sim 1$, respectively. It is observed that a good agreement is obtained between the two values set at all values of $\alpha, \beta, \theta_{d}$, and $D$, respectively. Thus the ability of the proposed method to obtain full-range measurements of the principal axis angle is confirmed.

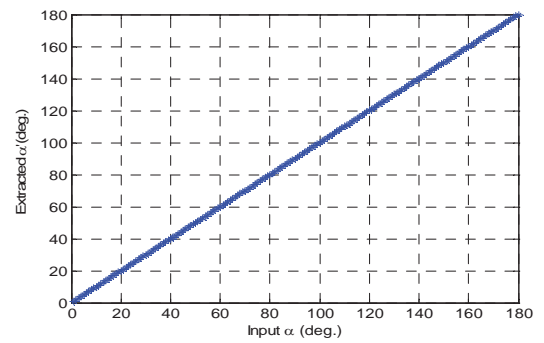

(a)

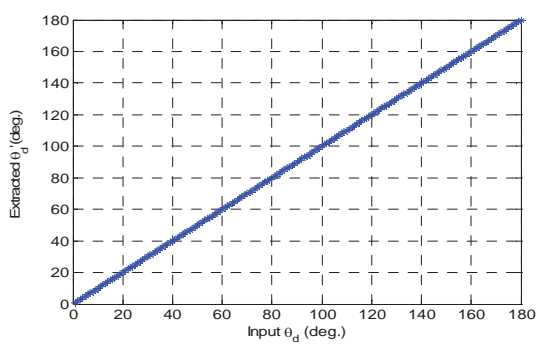

(c)

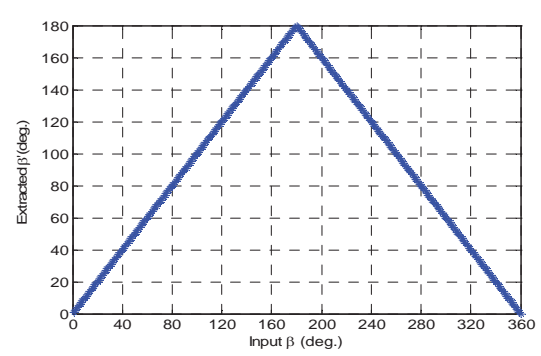

(b)

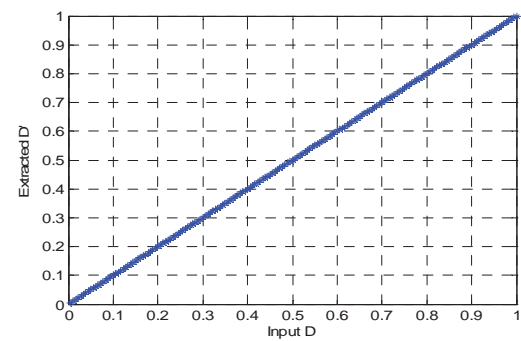

(d)

Fig. 1. Correlation between input value and extracted value of (a) principal axis angle $(\alpha) \&$ $\left(\alpha^{\prime}\right),(\mathrm{b})$ phase retardance $(\beta) \&\left(\beta^{\prime}\right),(\mathrm{c})$ diattenuation axis angle $(\theta \mathrm{d}) \&\left(\theta \mathrm{d}^{\prime}\right)$, and $(\mathrm{d})$ diattenuation (D) \& (D'), respectively.

Overall, the results presented in Figs. 1(a) $\sim$ (d) demonstrate that the proposed method yields full range measurements of all the linear birefringence / linear diattenuation parameters of interest other than the phase retardance, which is limited to the range $0 \sim 180^{\circ}$. Nonetheless, the proposed method enables the complete characterization of optical samples such as thin films, in which the phase retardance is limited to this restricted range due to their very thin thickness. 


\section{Experimental measurements and results}

Figure 2 presents a schematic illustration of the experimental setup used in this study to evaluate the practical feasibility of the proposed measurement method. Note that in this figure, $\mathrm{P}$ is a polarizer (GTH5M, Thorlabs Co.) and Q is a quarter-wave plate (QWP0-633-04-4-R10, CVI Co.). As shown, the polarizer and quarter-wave plate were used to produce linearly polarized light orientated at $0^{\circ}$, $45^{\circ}, 90^{\circ}$, and $135^{\circ}$ to the horizontal plane, respectively, and right-handed circularly polarized light. In addition, the slow axis or the diattenuation axis of the sample was set to various positions (i.e., $0^{\circ}$, $30^{\circ}, 60^{\circ}, 90^{\circ}, 120^{\circ}$, or $150^{\circ}$ ) during the measurement process using a rotary stage. In the experiments, the illuminating light was provided by a frequency-stable He-Ne laser (SL 02/2, SIOS Co.) with a central wavelength of $632.8 \mathrm{~nm}$. Finally, the output Stokes parameters were determined in accordance with the intensity measurements obtained using a commercial Stokes polarimeter (PAX5710, Thorlabs Co.).

If the sample has a diattenuation property, the output Stokes parameters can not be normalized, and thus additional steps must be taken to ensure that each of the five input lights has an identical intensity. In the present experiments, this is achieved by using a Neutral Density Filter (NDC-100C2, ONSET Co.) and a power meter detector (8842A, OPHIT Co.) positioned between the polarizer and the sample (see Fig. 2).

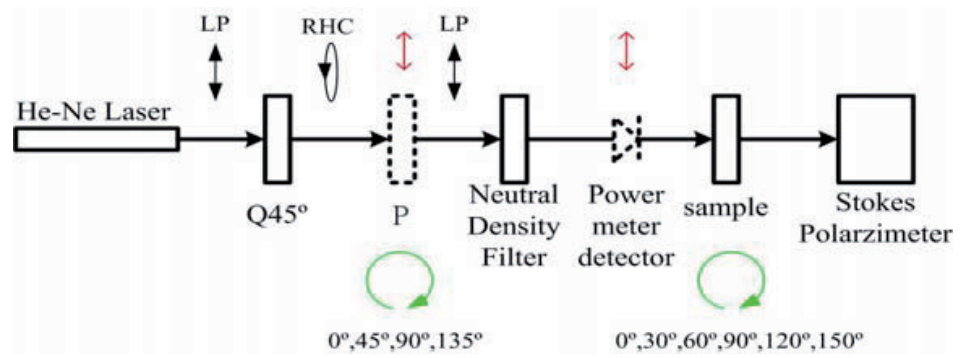

Fig. 2. Schematic illustration of experimental measurement system.

The sampling rate of polarimeter used in the present experiments of measuring the output Stokes parameters is 30 samples per second. Therefore, we used one hundred data points to calculate the standard deviation and the average data in experiments. The validity of the proposed measurement method was evaluated using four different optical samples, namely a quarter-wave plate (QWP0633-04-4-R10, CVI Co.), a polarizer (GTH5M, Thorlabs Co.), a second polarizer (LLC2-82-18S, OPTIMAX Co.) baked in an oven at a temperature of $150^{\circ} \mathrm{C}$ for 80 minutes, and a composite sample comprising of the quarter-wave plate and the baked polarizer, respectively. The quarter-wave plate and polarizer were chosen specifically to evaluate the performance of the proposed method in measuring the parameters of samples with low diattenuation and low birefringence, respectively.

\subsection{Quarter-wave plate as a sample}

Figs. 3(a) and (b) illustrate the experimental results obtained for the linear birefringence and linear diattenuation properties of the quarter-wave plate. In Fig. 3(a), the average standard deviations of the principal axis angle and phase retardance are found to be $0.03^{\circ}$ and $0.04^{\circ}$, respectively. In Fig. 3(b), it is observed that the extracted value of the diattenutation axis angle varies non-linearly with changes in the slow axis angle of the quarter-wave plate. Moreover, it can be seen that the value of the extracted diattentuation fluctuates notably with changes in the rotational position of the sample. 


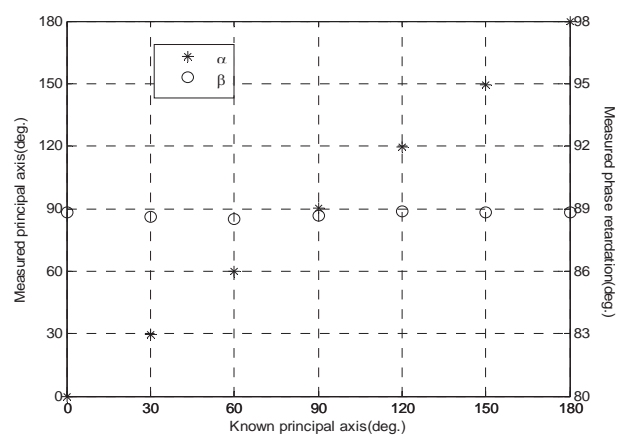

(a)

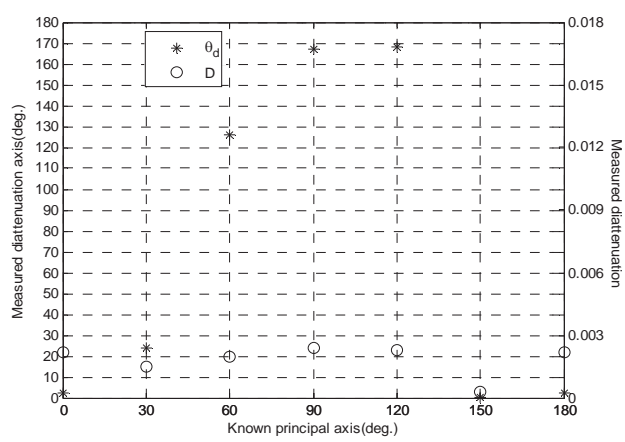

(b)

Fig. 3. Experimental results for (a) birefringence/ (b) diattenuation of quarter-wave plate.

\subsection{Polarizer as a sample}

Figures 4(a) and (b) present the experimental results obtained for the birefringence and diattentuation parameters of the polarizer. As expected, Fig. 4(a) shows that the diattenuation of the polarizer has a value equal to approximately 1 . Moreover, the average standard deviations of $\theta_{d}$ and $D$ are found to be around $0.03^{\circ}$ and $5.38 \times 10^{-5}$, respectively. Consequently, the extracted values of both birefringence parameters deviate significantly from the expected values for optical samples with low birefringence.

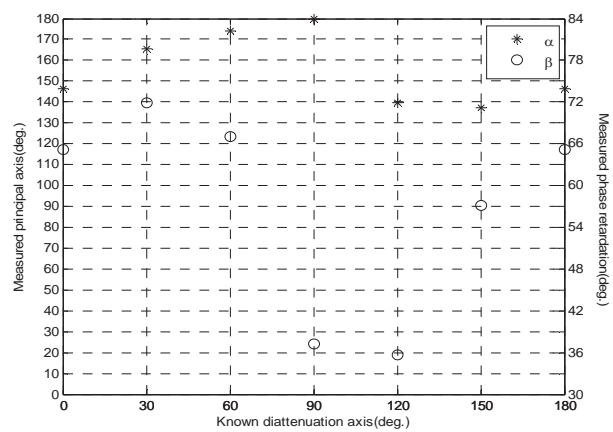

(a)

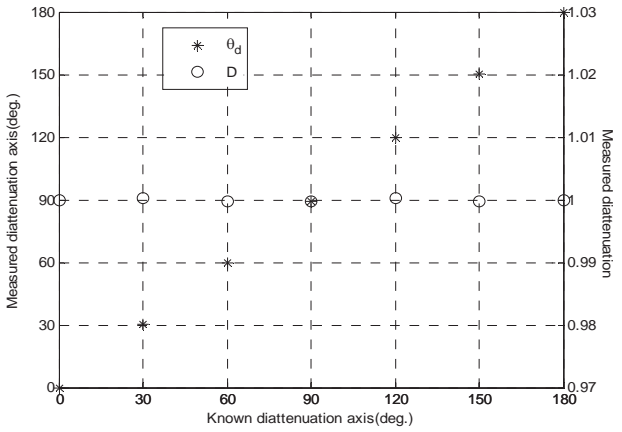

(b)

Fig. 4. Experimental results obtained for (a) birefringence/ (b) diattenuation of polarizer.

\subsection{Baked polarizer as a sample}

Figures 5(a) and (b) illustrate the experimental results obtained for the effective linear birefringence and linear diattenuation properties of the baked polarizer. The average measured values of the four optical parameters of baked polarizer with different diattenuation axis angles are summarized in Table 1. As expected, the diattenuation has a value of less than 1 since the prolonged exposure of the polarizer to a high-temperature environment causes the optical intensity of the input light to leak through one of the diattenuation axes. It is observed that the baked polarizer as mentioned before causes the polarizer to develop distinct birefringence properties and the retardance has a nonisotropic characteristic. Interestingly, it is also seen that the principal axis angle and the diattenuation axis angle do not coincide exactly with one another. 


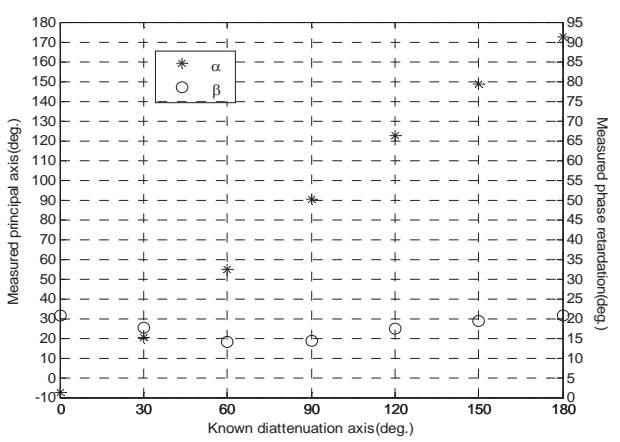

(a)

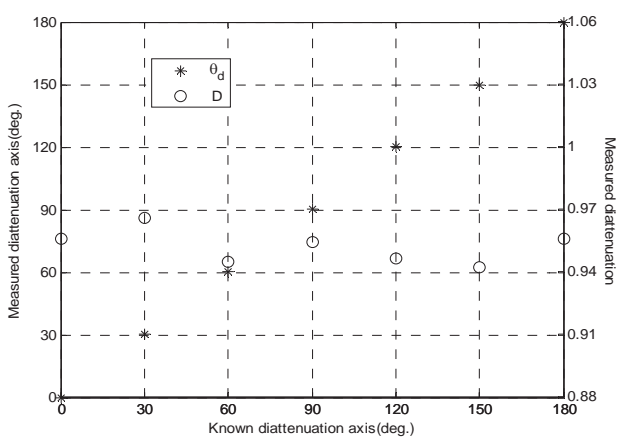

(b)

Fig. 5. Experimental results obtained for effective (a) birefringence/ (b) diattenuation of baked polarizer.

The results show that the sample has both birefringence and diattenuation. Thus, the ability of the measurement system to extract the characteristic parameters of samples with both birefringence and diattenuation is confirmed.

Table 1. Experimental data obtained for the effective birefringence and diattenuation of baked polarizer.

\begin{tabular}{|c|c|c|c|c|c|c|c|}
\hline Known diattenuation axis (deg.) & 0 & 30 & 60 & 90 & 120 & 150 & 180 \\
\hline$\alpha$ (deg.) & -7.71 & 20.02 & 54.51 & 90.52 & 122.42 & 148.57 & 172.27 \\
\hline$\beta$ (deg.) & 20.83 & 17.72 & 14.17 & 14.41 & 17.43 & 19.27 & 20.82 \\
\hline$\theta_{d}$ (deg.) & 0.09 & 30.15 & 60.23 & 90.08 & 120.08 & 149.97 & 179.92 \\
\hline$D$ & 0.956 & 0.966 & 0.945 & 0.954 & 0.947 & 0.942 & 0.956 \\
\hline
\end{tabular}

\section{Conclusions}

This study has proposed a technique based on a polarimeter and the Mueller matrix formulation for measuring the linear birefringence and linear diattenuation properties of an optical sample. The validity of the proposed approach has been demonstrated by measuring the principal axis angle $(\alpha)$, retardance $(\beta)$, diattenuation axis angle $\left(\theta_{d}\right)$, and diattenuation $(D)$ of a quarter-wave plate, a polarizer, a baked polarizer, and a composite sample comprising a quarter-wave plate and a baked polarizer, respectively. It has been shown that the proposed methodology enables the full-range measurement of the principal axis angle, diattenuation axis angle, and diattenuation, respectively. However, the dynamic measurement range of the retardance is limited to $0 \sim 180^{\circ}$. Nonetheless, the proposed method still enables the full characterization of samples such as thin films, in which the retardance is restricted to the range $0 \sim 180^{\circ}$ due to their very thin thickness. As compared to many of the optical parameter measurement schemes presented in the literature, the methodology proposed in this study does not require the birefringence and diattenuation axes of the sample to coincide. In addition, the linear birefringence and linear diattenuation parameters are decoupled in the analytical model. Thus, the birefringence of the optical sample can be evaluated without any prior knowledge of the diattenuation characteristics.

\section{Acknowledgements}

The authors gratefully acknowledge the financial support provided to this study by the National Science Council of Taiwan under grant NSC96-2628-E-006-005-MY3. 


\section{References}

1. R. A. Chipman, Opt. Eng. 28, 90-99 (1989).

2. J. P. McGuire, Jr., Ph.D. thesis, University of Alabama in Huntsville, (1990).

3. R. A. Chipman, Handbook of Optics (McGraw-Hill, 1995), Vol. 2, Chap. 22.

4. D. B. Chenault and R. A. Chipman, Polarimetry: Radar, Infrared, Visible, Ultraviolet, And XRay 1317, 263-278, (1990).

5. D. B. Chenault and R. A. Chipman, Opt. Lett. 17, 4223-4227 (1992).

6. D. B. Chenault and R. A. Chipman, Appl. Opt. 32, 3513-3519 (1993).

7. J. M. Bueno and P. Artal, Invest. Ophthalmol. Vis. Sci. 46, (2005).

8. J. M. Bueno and P. Artal, J. Mod. Opt. 55, 849-859 (2008).

9. M. Todorovic, S. L. Jiao, and L. V. Wang, Opt. Lett. 29, 2402-2404 (2004).

10. X. R. Huang and R.W. Knighton, Appl. Opt. 42, 5737-5743 (2003).

11. D. A. Higgins, D. A. VandenBout, J. Kerimo, and P. F. Barbara, J. Phys. Chem. 100, 1379413803 (1996).

12. A. L. Campillo and J. W. P. Hsu, J. Appl. Phys. 91, 646-651 (2002).

13. M. J. Fasolka, L. S. Goldner, J. Hwang, A. M. Urbas, P. Swager, T. DeRege, and E. L. Thomas, Phys. Rev. Lett. 90, 016107 (2003).

14. L. S. Goldner, M. J. Fasolka, S. Nougier, H. P. Nguyen, G. W. Bryant, J. Hwang, K. D. Weston, K. L. Beers, A. Urbas, and E. L. Thomas, Appl. Opt. 42, 3864-3881 (2003).

15. L. S. Goldner, M. J. Fasolka, and S. N. Goldie, Appl. of Scanned Probe Microscopy to Polymers 897, 65-84 (2005).

16. L. S. Goldner, S. N. Goldie, M. J. Fasolka, F. Renaldo, J. Hwang, and J. F. Douglas, Appl. Phys. Lett. 85, 1338-1340 (2004).

17. <http://www.meadowlark.com>.

18. I. C. Khoo and F. Simoni, Physics of Liquid Crystalline Materials (Gorden and Breach Science Publishers, 1991), Chap. 13.

19. L. Giudicotti and M. Brombin, Appl. Opt. 46, 2638-2647 (2007).

20. H. Dong, Y. D. Gong, V. Paulose, P. Shum, and M. Olivo, Opt. Exp. 17, 13017-13028 (2009). 